\title{
Hyperon-rich Matter in Neutron Stars
}

\author{
Jürgen Schaffner and Igor N. Mishustin* \\ The Niels Bohr Institute \\ Blegdamsvej 17 \\ DK-2100 Copenhagen
}

\begin{abstract}
We study the equation of state of hyperon-rich matter for neutron stars using an extended relativistic mean-field model. We take special care of the recently proposed non-linear behaviour of the vector field which gives a much better description of Dirac-Brückner calculations. The hyperon-hyperon interaction is also implemented by introducing additional meson exchanges. These new terms avoid the instability found at high densitites in previous works while keeping the excellent description for finite nuclear systems. We also demonstrate within the mean-field approach that the presence of hyperons inside neutron stars on one hand and the hyperon-hyperon interactions on the other hand make the onset of kaon condensation less favourable.
\end{abstract}

\section{INTRODUCTION}

Nuclear matter at high densities exhibits a new degree of freedom: strangeness, hyperons and possibly kaons, occur at a moderate density of about 2-3 times normal nuclear density in neutron star matter [1]. These new particles influence the properties of the equation of state of matter and the global properties of neutron stars. Usually the relativistic mean

\footnotetext{
*permanent address: The Kurchatov Institute Russian Research Center, Moscow 123182, Russia
} 
field (RMF) model is adopted for describing the equation of state with hyperons. But the standard approach suffers of several shortcomings: First, the parametrizations adjusted to reproduce the properties of nuclei fail at high densities due to some instabilities of the scalar selfinteraction (a possible solution to this problem has been suggested by Reinhard [2]). Second the equation of state is much stiffer compared to nonrelativistic or Dirac-Brückner calculations and the effective mass is much smaller $\left(m^{*} / m \approx 0.5-0.6\right)$ in the ground state. A possible way out is to introduce a quartic selfinteraction term for the vector field [3]. This model gives a good description of the properties of nuclei [4] and is in reasonable agreement with Dirac-Brückner calculations [5]. In addition the instability found in the standard approach disappears. The equation of state for neutron stars (without hyperons) is considerably softened [4]. Third the hyperon-hyperon interaction becomes important for hyperon-rich matter present in the dense interior of neutron stars (note that nearly equal amounts of hyperons and nucleons are predicted [1]). The standard RMF model is not suited to reproduce the strongly attractive hyperon-hyperon interaction seen in double $\Lambda$ hypernuclei. An improved Lagrangian incorporating an additional pair of (hidden) strange meson fields remedies the situation [6.77. These additional interaction terms have never been applied for the equation of state of neutron star matter before.

In this paper we study the equation of state of neutron star matter for representative parameter sets which cover more or less all presently availabe fits of the relativistic mean field model to the properties of finite nuclei. The model is extended to include hyperons in a controllable way by fitting the parameters to hypernuclear data. In addition the hyperonhyperon interaction is introduced through (hidden) strange meson exchanges.

When describing the properties of dense matter it is also necessary to study the possibility of pseudoscalar meson condensation. In particular, much attention has been paid in recent years to the kaon condensation in neutron stars [8]. Most recent calculations based on chiral perturbation theory [9, 10] show that kaon condensation may set in at densities only 3-4 times larger than normal nuclear density. Nevertheless, these calculations do not take into account the presence of hyperons which will already occupy a large fraction of matter 
when the kaons possibly start to condense. On the other side, the calculations including hyperons have not taken into account the possible kaon condensed phase. Only recently, some work has been started to incorporate the hyperon and kaon degrees of freedom at the same time [11,12]. In ref. [11] the chiral Lagrangian by Kaplan and Nelson [8] is used for the baryons and kaons. The hyperons have not been included explicitly as constituents of the ground state, they are only considered through particle-hole excitations induced by the p-wave kaon-hyperon interaction. In this approach kaon condensation is predicted around $3 \rho_{0}$. The other work [12] uses the standard relativistic mean field (RMF) approach for the baryon sector and the kaon-baryon interactions from the Kaplan-Nelson Lagrangian. It is shown that the critical density for kaon condensation is shifted to higher densities when hyperons are included $\left(\rho_{c}>4 \rho_{0}\right)$. It has been observed that the nucleon effective mass is shifted towards negative values at high densities due to the kaon condensate. The authors conclude that the RMF model breaks down at these densities and that one has to go beyond the mean-field level. Thus, two approaches using the same kaon-baryon interactions come to different conclusions. In view of the present interest we also include the kaons in our RMF model by using kaon-baryon interactions motivated by meson exchange models. We demonstrate that the additional (hidden) strange meson fields mentioned above make kaon condensation less favourable even at very high densities.

\section{THE EXTENDED RMF MODEL}

We start from the standard Lagrangian

$$
\begin{aligned}
\mathcal{L}= & \sum_{\mathrm{B}} \bar{\Psi}_{B}\left(i \gamma^{\mu} \partial_{\mu}-m_{B}\right) \Psi_{B}+\frac{1}{2} \partial^{\mu} \sigma \partial_{\mu} \sigma-U(\sigma) \\
& -\frac{1}{4} G^{\mu \nu} G_{\mu \nu}+\frac{1}{2} m_{\omega}^{2} V^{\mu} V_{\mu}-\frac{1}{4} \vec{B}^{\mu \nu} \vec{B}_{\mu \nu}+\frac{1}{2} m_{\rho}^{2} \vec{R}^{\mu} \vec{R}_{\mu} \\
& -\sum_{\mathrm{B}} g_{\sigma B} \bar{\Psi}_{B} \Psi_{B} \sigma-\sum_{\mathrm{B}} g_{\omega B} \bar{\Psi}_{B} \gamma^{\mu} \Psi_{B} V_{\mu}-\sum_{\mathrm{B}} g_{\rho B} \bar{\Psi}_{B} \gamma^{\mu} \vec{\tau}_{B} \Psi_{B} \vec{R}_{\mu}
\end{aligned}
$$

where the sum runs over all baryons of the baryon octet $\left(\mathrm{p}, \mathrm{n}, \Lambda, \Sigma^{+}, \Sigma^{0}, \Sigma^{-}, \Xi^{0}, \Xi^{-}\right)$. The term $U(\sigma)$ stands for the scalar selfinteraction 


$$
U(\sigma)=\frac{1}{2} m_{\sigma}^{2} \sigma^{2}+\frac{b}{3} \sigma^{3}+\frac{c}{4} \sigma^{4}
$$

introduced by Boguta [13] to get a correct compressibility of normal nuclear matter. The parameters of this Lagrangian have been fitted to the properties of finite nuclei [14,15]. It turned out that the best fits are obtained for the parameter sets with $c<0$. In this case the functional form (2) of the scalar field potential leads to an instability at high densities. Its traces are already seen in the nucleus ${ }^{12} \mathrm{C}[2]$. Hence, another stabilized functional form has been given by Reinhard [2] which eliminates the instability while keeping the good description of nuclei, especially the spin-orbit splitting. Alternatively, Bodmer proposed an additional selfinteraction term for the vector field [3] of the form

$$
\mathcal{L}_{V^{4}}=\frac{1}{4} d\left(V_{\mu} V^{\mu}\right)^{2}
$$

This modification leads to a nice agreement with Dirac-Brückner calculations at high densities [5]. The reason is that the vector field is then proportional to $\rho^{1 / 3}$ in contrast to the linear dependence in the standard model. The fits to the properties of nuclei are quite succesful and the instability due to the scalar selfinteraction vanishes [4].

The implementation of hyperons is straightforward. The corresponding new coupling constants have been fitted to hypernuclear properties [16]. It turns out that the two coupling constants of the $\Lambda\left(g_{\sigma \Lambda}\right.$ and $\left.g_{\omega \Lambda}\right)$ are strongly correlated because they are fixed by the depth of the $\Lambda$-potential

$$
U_{\Lambda}^{(N)}=g_{\sigma \Lambda} \sigma^{\text {eq. }}+g_{\omega \Lambda} V_{0}^{\text {eq. }}
$$

in saturated nuclear matter [17, 18]. Here $U_{i}^{(j)}$ denotes the potential depth of a baryon species $i$ in matter of baryon species $j$. Hence one can use for example $\mathrm{SU}(6)$-symmetry for the vector coupling constants

$$
\frac{1}{3} g_{\omega N}=\frac{1}{2} g_{\omega \Lambda}=\frac{1}{2} g_{\omega \Sigma}=g_{\omega \Xi}, \quad g_{\rho N}=\frac{1}{2} g_{\rho \Sigma}=g_{\rho \Xi}, \quad g_{\rho \Lambda}=0
$$

and fix the scalar coupling constants to the potential depth of the corresponding hyperon. Following [6,7] we choose 


$$
U_{\Lambda}^{(N)}=U_{\Sigma}^{(N)}=-30 \mathrm{MeV} \quad, \quad U_{\Xi}^{(N)}=-28 \mathrm{MeV}
$$

in accordance with the available hypernuclear data. This constitutes our model 1.

Nevertheless, these models are not able to reproduce the observed strongly attractive $\Lambda \Lambda$ interaction irrespectively of the chosen vector coupling constant. The situation can be remedied by introducing two additional meson fields, the scalar meson $f_{0}(975)$ (denoted as $\sigma^{*}$ in the following) and the vector meson $\phi(1020)$ with the masses given in parenthesis [6,7]. The corresponding Lagrangian reads

$$
\begin{aligned}
\mathcal{L}^{Y Y}= & \frac{1}{2}\left(\partial_{\nu} \sigma^{*} \partial^{\nu} \sigma^{*}-m_{\sigma^{*}}^{2} \sigma^{* 2}\right)-\frac{1}{4} S_{\mu \nu} S^{\mu \nu}+\frac{1}{2} m_{\phi}^{2} \phi_{\mu} \phi^{\mu} \\
& -\sum_{B} g_{\sigma^{*} B} \bar{\Psi}_{B} \Psi_{B} \sigma^{*}-\sum_{B} g_{\phi B} \bar{\Psi}_{B} \gamma_{\mu} \Psi_{B} \phi^{\mu}
\end{aligned}
$$

The vector coupling constants to the $\phi$-field are given by SU(6)-symmetry (see [7] for details)

$$
2 g_{\phi \Lambda}=2 g_{\phi \Sigma}=g_{\phi \Xi}=-\frac{2 \sqrt{2}}{3} g_{\omega N}, \quad g_{\phi N}=0
$$

The scalar coupling constants to the $\sigma^{*}$-field are fixed by the condition

$$
U_{\Xi}^{(\Xi)} \approx U_{\Lambda}^{(\Xi)} \approx 2 U_{\Xi}^{(\Lambda)} \approx 2 U_{\Lambda}^{(\Lambda)} \approx-40 \mathrm{MeV}
$$

which is motivated by the one-boson exchange model D of the Nijmegen group and the measured strong $\Lambda \Lambda$ interaction [7]. Note that the nucleons are not coupled to these new fields. In the following we denote the extended model with hyperon-hyperon interactions as model 2 .

\section{NEUTRON STAR MATTER WITH HYPERONS}

The equation of state for neutron star matter is derived by standard methods (see e.g. ref. [1] for the standard RMF approach). The equations of motion for the meson fields in uniform matter are given by

$$
m_{\sigma}^{2} \sigma+\frac{\partial}{\partial \sigma} U(\sigma)=\sum_{\mathrm{B}} g_{\sigma B} \rho_{S}^{(B)}
$$




$$
\begin{aligned}
m_{\sigma^{*}}^{2} \sigma^{*} & =\sum_{\mathrm{B}} g_{\sigma^{*} B} \rho_{S}^{(B)} \\
m_{\omega}^{2} V_{0}+d V_{0}^{3} & =\sum_{\mathrm{B}} g_{\omega B} \rho_{V}^{(B)} \\
m_{\rho}^{2} R_{0,0} & =\sum_{\mathrm{B}} g_{\rho B} \tau_{3}^{B} \rho_{V}^{(B)} \\
m_{\phi}^{2} \phi_{0} & =\sum_{\mathrm{B}} g_{\phi B} \rho_{V}^{(B)}
\end{aligned} .
$$

where $\rho_{S}$ and $\rho_{V}$ denote the scalar and vector densities, respectively. The equations can be solved for a given total baryon density $\rho_{B}$ and charge density $\rho_{c}$ including the contributions from the free electrons and muons

$$
\begin{aligned}
\rho_{B} & =\sum_{\mathrm{B}} \rho_{V}^{(B)} \\
\rho_{c} & =\sum_{\mathrm{B}} q_{B} \rho_{V}^{(B)}+\sum_{l=e, \mu} q_{l} \rho_{l}=0
\end{aligned}
$$

where $q_{i}$ stands for the electric charge of a species $i$. In $\beta$-equilibrium the chemical potentials of the particles are related to each other by

$$
\mu_{i}=B_{i} \cdot \mu_{B}+q_{i} \cdot \mu_{e}
$$

where $B_{i}$ is the baryon number of a species $i$. This means that all reactions which conserve charge and baryon number are allowed, as e.g.

$$
\mathrm{n}+\mathrm{n} \rightarrow \Lambda+\mathrm{n} \quad, \quad \Lambda+\Lambda \rightarrow \Xi^{-}+\mathrm{p} \quad, \quad \ldots
$$

Since we consider neutron stars in a long time scale, the strangeness quantum number is not constrained and the net strangeness is determined by the condition of $\beta$-equilibrium. The above equations fixes already the fields and the composition of neutron star matter. The energy and pressure density can be derived from the grand canonical potential or the energymomentum tensor in a standard way (see e.g. [1]) and the generalization to the additional fields is straightforward:

$$
\begin{aligned}
\epsilon= & \frac{1}{2} m_{\sigma}^{2} \sigma^{2}+\frac{b}{3} \sigma^{3}+\frac{c}{4} \sigma^{4}+\frac{1}{2} m_{\sigma^{*}}^{2} \sigma^{* 2}+\frac{1}{2} m_{\omega}^{2} V_{0}^{2}+\frac{3}{4} d V_{0}^{4} \\
& +\frac{1}{2} m_{\rho}^{2} R_{0,0}^{2}+\frac{1}{2} m_{\phi}^{2} \phi_{0}^{2}+\sum_{i=B, l} \frac{\nu_{i}}{\left(2 \pi^{3}\right)} \int_{0}^{k_{F}^{i}} d^{3} k \sqrt{k^{2}+m_{i}^{* 2}}
\end{aligned}
$$




$$
\begin{aligned}
P= & -\frac{1}{2} m_{\sigma}^{2} \sigma^{2}-\frac{b}{3} \sigma^{3}-\frac{c}{4} \sigma^{4}-\frac{1}{2} m_{\sigma^{*}}^{2} \sigma^{* 2}+\frac{1}{2} m_{\omega}^{2} V_{0}^{2}+\frac{1}{4} d V_{0}^{4} \\
& +\frac{1}{2} m_{\rho}^{2} R_{0,0}^{2}+\frac{1}{2} m_{\phi}^{2} \phi_{0}^{2}+\sum_{i=B, l} \frac{\nu_{i}}{\left(2 \pi^{3}\right)} \int_{0}^{k_{F}^{i}} d^{3} k \frac{k^{2}}{\sqrt{k^{2}+m_{i}^{* 2}}}
\end{aligned}
$$

where

$$
m_{B}^{*}=m_{B}+g_{\sigma B} \sigma+g_{\sigma^{*} B} \sigma^{*}
$$

is the effective mass of the baryon $B$ (for leptons the on-shell mass is taken).

In the following we study the composition of neutron star matter for the various parameter sets obtained from fits to finite nuclei. First we take the sets NL-Z (which is set NL-1 with a more consistent center-of-mass correction [14]), NL-SH [15] for the Boguta-model and the sets PL-40 and PL-Z [2] with the stabilized scalar functional. The corresponding coupling constants are listed in table 1 . The first parameter set shows a well known instability at high densities [2] which appears in neutron star matter around $\rho \approx 0.5 \mathrm{fm}^{-3}$. At this point the effective mass of the nucleon gets negative due to the presence of the hyperons. For higher density no solution can be found. Negative effective nucleon masses appear also for set NL-SH at a density of $\rho \approx 0.8 \mathrm{fm}^{-3}$ for model 1 and at $1.0 \mathrm{fm}^{-3}$ for model 2 . The same holds for the case with the stabilized functional forms, sets PL-Z and PL-40 [2], in model 1 and 2 around a similar density region. Note, that this happens even without the presence of a kaon condensate as found in [12]. One might wonder about the fact, that the early occurence of negative masses found here has not been seen in [12]. The explanation is simple: The standard parameter sets from fits to finite nuclei always favour a stiff equation of state to get a correct spin-orbit splitting which inhibits this instability as the fields are growing fast with the density. Fits to nuclear matter only can lead to a rather soft equation of state which avoids the instability due to a moderate rise of the fields. Nevertheless, the latter parametrizations are not able to describe the strong spin-orbit splitting seen in finite nuclei (for a detailed discussion see [14]) and therefore can not be considered as realistic. In the following we extended our calculation to densities beyond the instability, by taking always the absolut value $\left|m_{N}^{*}\right|$ of the effective mass (it is clear, that a much more refined 
procedure is necessary to treat the problem of negative effective masses, i.e. going beyond the mean-field appoximation).

The behaviour for lower densities is quite similar in all these models. Fig. 1 shows as an example the composition of neutron star matter for the set PL-Z and model 2. The proton fraction raises rapidly and reaches maximum values over $20 \%$ around $2-3 \rho_{0}$. At this density, the hyperons, first $\Lambda$ 's and $\Sigma^{-}$'s then $\Xi^{-}$'s, appear abundantly and the lepton fraction is considerably reduced. When the other hyperons are present at densities of $3-4 \rho_{0}$ the number of $\Lambda$ 's even exceeds the number of neutrons, so that the dense interior resembles more a hyperon star than a neutron star [1]. Also the population of leptons gets negligibly low, as the electrochemical potential drops instead of growing. We have also checked for the occurence of $\Omega^{-}$which do not contribute in any of our calulations.

The situation for higher densities differs for the various parameter sets mainly due to the instability mentioned above. Taking the absolute value of the effective nucleon mass still no solution can be found for the sets NL-Z and NL-SH above the critical density for model 1. For the sets PL-Z and PL-40 we found a solution and the neutron star matter becomes pure hyperon matter dominated by $\Lambda$ 's and no nucleons appear above the critical density. Also the electrochemical potential changes sign and positrons and antimuons appear instead of electrons and muons. When turning on the hyperon-hyperon interactions (model 2) the hyperons feel at these high densities effectively an additional repulsion. Hence, nucleons are now present but still considerably less abundant than hyperons. Now a solution for set NL-SH can be also found. Nevertheless, these results should be taken with some care as we do not treat the problem of negative effective masses consistently.

Now we turn our discussion to the case of models with vector selfinteractions. Two sets, named TM1 and TM2, exist so far in the literature [4] (see also table 1). It has already been found that the behaviour of the nuclear equation of state follows more nicely the trends of relativistic Brückner-Hartree-Fock calculations and that the maximum mass of a neutron star is reduced [4]. Fig. 2 shows now the composition of neutron star matter for the set TM1 with hyperons and with hyperon-hyperon interactions (model 2). Up to 
the maximum density considered, here $10 \rho_{0}$, all effective masses remain positive and no instability occurs. The behaviour at moderate densities is quite close to that of the previous parametrizations: The proton fraction has a plateau at $2-4 \rho_{0}$ and exceeds $20 \%$ which allows for the direct URCA process and a rapid cooling of the neutron star [19]. Hyperons, first $\Lambda^{\prime}$ 's and $\Sigma^{-}$'s, appear at $2 \rho_{0}$, then $\Xi^{-}$'s are populated already at $3 \rho_{0}$. The number of electrons and muons has a maximum here. The muons vanish when all the hyperons have been settled at $\rho \approx 0.85 \mathrm{fm}^{-3}$ while the electrons remain on the level of $2 \%$. The fractions of all baryons show a tendency towards saturation, they reach asymptotically similar values of $8-15 \%$ which corresponds approximately to spin- and isospin-saturated matter. In the ideal case all baryons fractions would be the same due to the same spin-degeneracy factors.

Switching off the hyperon-hyperon interactions, i.e. going from model 2 to model 1, we see again that negative effective masses occur at $\rho=1.3 \mathrm{fm}^{-3}$ for set TM1 and at $\rho=0.95 \mathrm{fm}^{-3}$ for set TM2 due to the missing additional repulsive force for the hyperons. Nevertheless, the main population pattern is not changed considerably below $\rho=0.6 \mathrm{fm}^{-3}$ when going from model 2 to model 1, but the number of hyperons, especially $\Lambda$ 's, are reduced at higher densities. Also the leptons vanishes for set TM1, but for the set TM2 positrons appear again, as the electrochemical potential changes sign above the occurrence of the instability. These results demonstrate the importance of the additional exchanges of $\sigma^{*}$ and $\phi$ mesons. An additional repulsion is needed at higher densities to stabilize neutron star matter.

The effective masses defined in (16) are plotted in fig. 3 for the various baryons for set TM1 and model 2. The effective mass of the nucleons reaches rapidly very small values and then saturates at higher densities never reaching negative values. For hyperons the behaviour is quite similar but less pronounced as their coupling constants to the $\sigma$-field are smaller than for nucleons. Nevertheless, the combined effect of the $\sigma$ and $\sigma^{*}$ fields results in a rather constant gap between the effective masses over the whole density range shown. Note that the nucleons do not couple to the (hidden) strange scalar field $\sigma^{*}$. The overall small masses of the baryons at very high densities indicate that the neutron star matter approaches isospin-saturated matter with equal abundances, as can be seen in fig. 2 . 
The pure scalar and vector field potentials are shown in fig. 4 (they have to be multiplied with the corresponding coupling constants given in table 1 to get the potential for a baryon species). In addition, we have also plotted the electrochemical potential. It reaches a maximum value of about $200 \mathrm{MeV}$ around $\rho \approx 2-3 \rho_{0}$ and gets then consideraly lower at high densities instead of growing steadily. This behaviour is due to the onset of negatively charged hyperons $\left(\Sigma^{-}\right.$and $\left.\Xi^{-}\right)$. The vector potential exhibits initially a linear rise which then slows down due to the vector selfinteraction and the onset of hyperons at $\rho \approx 0.3 \mathrm{fm}^{-3}$. At the same density the (hidden) strange fields are developed and reach quite high values for very dense matter. The $\phi$-field shows a rather linear behaviour with density while the scalar fields seems to saturate at high densities. The isovector potential coming from $\rho$-exchange is quite small over the whole density range and never exceeds $-60 \mathrm{MeV}$ for the nucleons. The other potentials are one order of magnitude higher and have opposite signs. That also indicates a large cancelation between scalar and vector terms. The fields get so strong, that, for example, a $10 \%$ change of one coupling constant can alter the potential up to $100 \mathrm{MeV}$ ! Therefore, it is very important to fine tune the scalar coupling constants according to the potential depth (see eqs. (6) and (91)). This has to be kept in mind for the forthcoming discussion of the properties of kaons in dense neutron star matter.

The equation of state, the pressure density versus the energy density is given in fig. 5 for all parameter sets using model 2. For $\epsilon<800 \mathrm{MeV} \cdot \mathrm{fm}^{-3}$ there exist mainly two bunches of curves. The parameter sets without vector selfinteraction are located in the upper branch. The sets TM1 and TM2 with vector selfinteraction are softer, i.e. they have a lower pressure for a given energy density and are therefore constituting the lower branch of curves. For higher energy densities, set TM2 shows to be stiffer than set TM1. For the upper branch one recognizes kinks in the curves which are due to the instability discussed above (i.e. negative effective masses). For model 1 (not shown), one sees a very pronounced jump in the curves due to the occurence of this instability. The equation of state gets then considerably softened reaching very low pressures. But for model 2 the jump is much less pronounced and even vanishes for the sets with vector selfinteraction. Another difference between model 1 and 
model 2 is the slight softening of the equation of state for medium densities which is due to the attractive hyperon-hyperon interaction mediated by the $\sigma^{*}$-meson exchange. For very high densities model 2 gets stiffer than model 1, because the contribution from the repulsive $\phi$-field overwhelms now the one coming from the attractive $\sigma^{*}$-field which is saturating at very high densities. In addition the stiffest possible equation of state $\epsilon=p$ is also drawn. The causality condition $\partial p / \partial \epsilon \leq 1$ is fulfilled by all sets, so that the speed of sound remains lower than the velocity of light. The microscopic stability condition $\partial p / \partial \epsilon \geq 0$ is also satisfied except for the instability region.

Finally, we want to study possible extensions of the RMF approach including other mesons. It is well known that pseudoscalar and pseudovector mesons do not contribute in the mean-field approximation. The same holds for all the kaon resonances as they couple offdiagonally. Tensor mesons also vanishes in infinite matter. Hence, the only remaining meson which can be added to the present Lagrangian is the scalar isovector meson $a_{0}(980)$ (the $\delta$-meson). To our knowledge this meson has been not considered so far for the equation of state of neutron stars. Fits to the properties of nuclei seems to indicate that its contribution are negligible for the discussion about binding energy, radius and surface thickness of stable nuclei [14]. Nevertheless, it might be important for very asymmetric systems. For the discussion in the next section it is quite useful to study the influence of this meson for the properties of neutron star matter. We implement the $\delta$-meson in the standard way and choose the coupling constant from the Bonn-model $\left(g_{\delta N}=5.95\right.$ [20]). The other coupling constants are scaled according to the isospin of the corresponding baryon $\left(2 g_{\delta N}=g_{\delta \Sigma}=\right.$ $\left.2 g_{\delta \Xi}, g_{\delta \Lambda}=0\right)$. As seen in fig. 4 the contribution from the vector isovector meson $\rho$ is quite negligible compared to the other fields. Therefore, we expect only minor changes when introducing the scalar isovector meson $\delta$. Indeed, the equation of state with the $\delta$-meson gets only a little bit stiffer for lower density regions. For higher densities symmetric matter is reached also and the equation of state approaches the one without the $\delta$-meson because the contribution of this meson simply vanishes in symmetric matter. The baryon composition shows some small changes for densities lower than $\rho<3 \rho_{0}$. As the $\delta$-field is repulsive for 
the protons, but attractive for the $\Sigma^{-}$'s, the former ones are a little bit suppressed while the latter ones appear slightly earlier. These effects reduce the number of electrons and the maximum chemical potential is now about $150 \mathrm{MeV}$ instead of $200 \mathrm{MeV}$. The meson fields do not change when the $\delta$-field is introduced. The most pronounced effect, despite of the lower electrochemical potential, is the change in the effective masses of the baryons which are plotted in fig. 6 . In contrast to fig. 3 every baryon has now a different effective mass. The biggest effect is seen for the $\Sigma^{-}$due to its large coupling constant to the $\delta$-field. The effective mass of the $\Sigma^{-}$can even be lower than that of the $\Lambda$. The neutron has a significantly lower effective mass than that of the proton. For high densities it can be a relative difference of a factor of two! The absolute difference can be as high as $120 \mathrm{MeV}$. Nevertheless, the potential coming from the $\delta$-field never exceeds $70 \mathrm{MeV}$ for the nucleons and is therefore negligible compared to the rather high potential terms coming from the isoscalar fields (see fig. 4).

\section{KAON INTERACTION IN THE RMF APPROACH}

The kaon-nucleon interactions has been studied recently within different approaches including the Bonn model [21], the Nambu-Jona-Lasinio model [22] and chiral perturbation theory [23]. Antikaons in dense nuclear matter are of special interest because they can form a condensate [8] leading to a considerable softening of the equation of state and a reduction of the maximum mass of neutron stars [9]. But this issue is still controversial. For instance rather strong nonlinear behaviour has been found by extrapolating KN-interactions derived from KN-scattering to dense nuclear matter in the NJL-model [22] where no hints for kaon condensation is seen. Applying the Bonn interaction scheme within the simple RMF approximation also leads a strong nonlinear density dependance disfavouring antikaon condensation [24]. Still, chiral perturbation theory gives a rather robust prediction for the onset of antikaon condensation at $\rho_{c} \approx 3-4 \rho_{0}$ [10].

We highly appreciate many efforts devoted to the consistent approach to kaon conden-

sation based on the chiral perturbation theory [10,23],25]. But in our opinion this approach 
has principal difficulties which may not be overcome in the near future. The main problem is that simple chiral models do not describe correctly the saturation properties of nuclear matter. By this reason the parameters used for the calculation of the kaon energy (coupling constants, effective masses, etc.) are not consistent with the properties of the background nuclear matter. Another and maybe related problem is that only linear terms in the baryon density are under control in the chiral approach. Therefore, all nonlinear terms are dropped out. In particular, the scalar density $\rho_{s}$ is identified with the baryon (vector) density $\rho$ of the matter. This approximation certainly fails at high densities (for instance at $\rho \approx 3 \rho_{0}$ the difference can be about $80 \%$ [2]). Already using the scalar density, as it is dictated by Lorentz invariance, shifts the kaon condensation treshold to densities above $5 \rho_{0}$ [24,26]. In ref. [24] it was also shown that many-body forces associated with the selfinteraction of the meson fields disfavour kaon condensation even more. By these reasons we use a more phenomenological approach based on the one-boson exchange model in the present paper.

As it is well known, there exists an important difference between $\mathrm{K}^{+} \mathrm{N}$ and $\mathrm{K}^{-} \mathrm{N}$ interaction. In contrast to $\mathrm{K}^{+} \mathrm{N}$-scattering the situation with $\mathrm{K}^{-} \mathrm{N}$-interactions is more complicated due to the existence of the $\Lambda(1405)$-resonance just below treshold. This makes the interpretation of the available $\mathrm{K}^{-} \mathrm{N}$-scattering and $\mathrm{K}^{-}$-atomic data less transparent. Recently an improved fit of $\mathrm{K}^{-}$-atomic data was carried out assuming a nonlinear density dependence of the effective $t$-matrix [27]. It has been shown that the real part of the antikaon optical potential can be as attractive as

$$
U_{\mathrm{opt}}^{\bar{K}} \approx-200 \pm 20 \mathrm{MeV}
$$

at normal nuclear matter density while being slightly repulsive at very low densities. This is in accordance with the low density theorem and $\mathrm{K}^{-}$p-scattering and effected by the $\Lambda(1405)$ resonance. Also another family of solutions have been found with a moderate potential depth around $-50 \mathrm{MeV}$. Note that also the standard linear extrapolation gives only values of about $-85 \mathrm{MeV}$ [27]. These latter two solutions do not fulfill the low density theorem, i.e. getting repulsive at low densities. There exist some hints that the $\Lambda(1405)$ is a quasi-bound state 
in the t-channel [28,21]. It is not surprising then that the $\mathrm{K}^{-} \mathrm{N}$-scattering data can be explained by a simple vector meson exchange [28] where the effects of this resonance come out automatically. This resonance seems to be less important in dense matter when the kaon mass is shifted down below $m_{\Lambda(1405)}-m_{N} \approx 465 \mathrm{MeV}$. In ref. [29] a separable potential was applied for the $\mathrm{K}^{-} \mathrm{p}$-interaction for finite density. Indeed, it was found that the mass of the $\Lambda(1405)$ is shifted upwards and exceeds the $\mathrm{K}^{-} \mathrm{p}$ threshold already at densities of about $\rho \approx 0.4 \rho_{0}$. In this case the use of mean-field potentials may be justified. Most recently, the $\Lambda(1405)$ has been also included in the chiral approach [25]. No effect has been found for the onset of kaon condensation [25], but note that the $\Lambda(1405)$ has been only implemented as a new field contrary to the conclusion drawn in [28,21]. In a recent preprint the coupled channel analysis of Siegel and Weise [28 has been applied for chiral perturbation theory [30]. The coupled channel formalism automatically generates the $\Lambda(1405)$ and succesfully describes the low energy $\mathrm{K}^{-} \mathrm{p}$-scattering data.

In our previous work [24] we have conjectured an important role which hyperons should play in determing the threshold of kaon condensation. Here we present the calculations which confirm these qualitative estimates. Recently, the effects of the presence of hyperons has been studied also in ref. [12]. The authors use the RMF model with scalar selfinteractions for the baryon sector and the chiral approach of Kaplan and Nelson [8] for the kaon-baryon interaction. It has been shown that the presence of hyperons, particular the $\Sigma^{-}$, shifts the threshold for $\mathrm{K}^{-}$condensation by several units of $\rho_{0}$. Note, however, that Ellis et al. do not take into account the terms which ensure that the $\Sigma$-term changes sign at low densities, as it should [10]. These additional terms will shift the onset of kaon condensation to even higher densities.

In the following we adopt the meson-exchange picture for the KN-interaction simply because we use it also for parametrizing the baryon interactions. By analogy to the Bonn model [21] the simplest kaon-meson Lagrangian can be written as

$$
\mathcal{L}_{K}=\partial_{\mu} \bar{K} \partial^{\mu} K-m_{K}^{2} \bar{K} K-g_{\sigma K} m_{K} \bar{K} K \sigma-g_{\sigma^{*} K} m_{K} \bar{K} K \sigma^{*}
$$




$$
-g_{\omega K} \bar{K} i \overleftrightarrow{\partial_{\mu}} K V^{\mu}-g_{\rho K} \bar{K} \vec{\tau}_{K} i \overleftrightarrow{\partial_{\mu}} K \vec{R}^{\mu}-g_{\phi K} \bar{K} i \overleftrightarrow{\partial_{\mu}} K \phi^{\mu}
$$

However, it is easy to see that this coupling scheme destroys the gauge invariance of the vector interactions represented by the equation

$$
\partial_{\mu} V^{\mu}=0
$$

From the equation of motion for the vector field $V^{\mu}$ one has

$$
\partial_{\mu} F^{\mu \nu}+m_{\omega}^{2} V^{\nu}=\sum_{\mathrm{B}} g_{\omega B} \bar{\Psi}_{B} \gamma^{\nu} \Psi_{B}+g_{\omega K}\left(\bar{K} i \stackrel{\leftrightarrow}{\partial_{\nu}} K\right)
$$

and after applying a partial derivative on both sides

$$
m_{\omega} \partial_{\nu} V^{\nu}=\sum_{\mathrm{B}} g_{\omega B} \partial_{\nu}\left(\bar{\Psi}_{B} \gamma^{\nu} \Psi_{B}\right)+g_{\omega K} \partial^{\nu}\left(\bar{K} i \stackrel{\leftrightarrow}{\partial_{\nu}} K\right)
$$

(the vector selfinteraction term is omitted here because it is purely phenomenological and the following arguments do not hold for it). The first term on the right hand side vanishes due to the baryon number conservation. The second term would also vanish for a free kaon, but it does not in the medium. Indeed, the conserved kaon current from the Lagrangian (18) reads

$$
\begin{aligned}
j_{\mu}^{K} & =i\left(\bar{K} \frac{\partial \mathcal{L}}{\partial^{\mu} \bar{K}}-\frac{\partial \mathcal{L}}{\partial^{\mu} K} K\right) \\
& =\bar{K} i \partial_{\mu} K-\left(i \partial_{\mu} \bar{K}\right) K+2 \bar{K} K\left(g_{\omega K} V_{\mu}+g_{\rho K} \vec{\tau} \vec{R}_{\mu}+g_{\phi K} \phi_{\mu}\right)
\end{aligned}
$$

so that this term does not vanish if the expectation value of the vector fields are nonzero. The situation can be corrected by introducing the additional terms

$$
\mathcal{L}_{K}^{\prime}=\mathcal{L}_{K}+\left(g_{\omega K} V_{\mu}+g_{\rho K} \vec{\tau} \vec{R}_{\mu}+g_{\phi K} \phi_{\mu}\right)^{2} \bar{K} K
$$

The kaon-meson Lagrangian can now be written as

$$
\mathcal{L}_{K}^{\prime}=D_{\mu}^{*} \bar{K} D^{\mu} K-m_{K}^{2} \bar{K} K-g_{\sigma K} m_{K} \bar{K} K \sigma-g_{\sigma^{*} K} m_{K} \bar{K} K \sigma^{*}
$$

with the covariant derivative 


$$
D_{\mu}=\partial_{\mu}+i g_{\omega K} V_{\mu}+i g_{\rho K} \vec{\tau} \vec{R}_{\mu}+i g_{\phi K} \phi_{\mu}
$$

The equation of motion for kaons in uniform matter then reads

$$
\begin{aligned}
\left\{\partial_{\mu} \partial^{\mu}+m_{K}^{2}\right. & +g_{\sigma K} \sigma m_{K}+g_{\sigma^{*} K} \sigma^{*} m_{K}+2\left(g_{\omega K} V_{0}+g_{\rho K} \tau_{0} R_{0,0}+g_{\phi K} \phi_{0}\right) i \partial^{\mu} \\
& \left.-\left(g_{\omega K} V_{0}+g_{\rho K} \tau_{0} R_{0,0}+g_{\phi K} \phi_{0}\right)^{2}\right\} K=0
\end{aligned}
$$

The coupling constants to the vector mesons are chosen from the $\mathrm{SU}(3)$-relations

$$
2 g_{\omega K}=2 g_{\rho K}=\sqrt{2} g_{\phi K}=g_{\pi \pi \rho}=6.04
$$

Decomposing the kaon field into plane waves one ontains the following dispersion relation for kaons (upper sign) and antikaons (lower sign) in uniform matter composed of nucleons and hyperons

$$
\omega_{K, \bar{K}}=\sqrt{m_{K}^{2}+m_{K}\left(g_{\sigma K} \sigma+g_{\sigma^{*} K} \sigma^{*}\right)+k^{2}} \pm\left(g_{\omega K} V_{0}+g_{\phi K} \phi_{0}+g_{\rho K} R_{0,0}\right) \quad .
$$

Note that due to the additional terms (23) the vector term appears linearly in the kaon energy.

The coupling constant $g_{\sigma K}$ could be taken from the Bonn model [21] but here we adopt another prescription. We want to fix it to the value of the potential depth of kaons in the nuclear medium to study the strongly attractive potential seen in kaonic atoms [27]. The optical potential in symmetric nuclear matter $\left(R_{0,0}=0, \phi_{0}=0\right.$, no hyperons, $\left.k=0\right)$ is in our approach given by comparison with eq. (26)

$$
2 \mu_{K N} U_{\mathrm{opt}}^{\bar{K}}=g_{\sigma K} \sigma m_{K}-2 g_{\omega K} \omega_{\bar{K}} V_{0}-\left(g_{\omega K} V_{0}\right)^{2}
$$

where $\mu_{K N}$ is the reduced mass of the $\mathrm{K}^{-} \mathrm{N}$-system. One notices that in general the optical potential and the energy shift (relative to the free mass) of the (anti)kaon do not coincide. In addition, the right hand side of eq. (29) shows a nonlinear density dependance (see also [27]). At normal nuclear density the in-medium energy shift of the antikaon is about $20 \%$ less than the corresponding optical potential! In the following we study two possibilities: 
we adjust the scalar coupling constant to $U_{\mathrm{opt}}^{\bar{K}}=-120 \mathrm{MeV}$, as an upper bound for the standard fit and the lower family of solutions found in [27], and to $U_{\mathrm{opt}}^{\bar{K}}=-200 \mathrm{MeV}$ for the second family of solutions.

For the $\bar{K} N$ case the low density theorem is not fulfilled due to the presence of the $\Lambda(1405)$ resonance (in principle one has to use a coupled-channel formalism to get the correct scattering length which is beyond the scope of this paper). We argue here that the repulsive regime at very low density is of no consequence for the evaluation of $\mathrm{K}^{-}$nuclear interaction at high densities as pointed out in 27,29.

The onset of (s-wave) kaon condensation is determined by the condition

$$
-\mu_{e}=\mu_{K^{-}}=\omega_{K^{-}}(k=0)
$$

When calculating $\mu_{K^{-}}$we have also taken into account the contribution from the (hidden) strange meson fields $\sigma^{*}$ and $\phi_{0}$ according to eq. (28). The coupling constant to the $\sigma^{*}$ field has been taken from $f_{0}$-decay [31] (note that this value is obtaint despite of the fact that the mass of the $f_{0}$ is lower than the $K \bar{K}$ treshold which constitutes another source of uncertainty). While the $\sigma^{*}$-field is attractive, the $\phi$-field is repulsive for antikaons. The isovector field is also repulsive for the $\mathrm{K}^{-}$as neutrons (and also $\Sigma^{-}$) are more abundant than protons $\left(\Sigma^{+}\right)$. As seen in fig. 5 the electrochemical potential decreases for higher densities. The relativistic effects, the reduced electrochemical potential, and the presence of the $\phi$ - and the $\rho$-meson fields make the kaon condensation less favourable for strangeness-rich matter.

In fig. 7 we plot $\omega_{K^{-}}-\mu_{K^{-}}$as a function of baryon density for model 2 and the various parameter sets considered. The upper curves correspond to $U_{\mathrm{opt}}^{\bar{K}}=-120 \mathrm{MeV}$ whereas the lower curves to $U_{\mathrm{opt}}^{\bar{K}}=-200 \mathrm{MeV}$. Below $2 \rho_{0}$ the curves drop rather steep and linear. After the appearance of the hyperons, the slope of the curves changes dramatically and they even turn upward again. Antikaon condensation is only possible if the curves crosses zero. This does not happen at all for the upper curves and only occurs for set PL-40 (but in the unstable regime with negative effective masses!) and for set TM1 among the lower curves. Note that the two sets TM1 and TM2, which do not get negative effective masses and might 
be the only two reliable calculations, give different predictions for the possible existence of a kaon condensed phase. While the former one reaches zero at $\rho \approx 6 \rho_{0}$, the latter one is at all densities at least $60 \mathrm{MeV}$ above the critical value.

For the case of $U_{\mathrm{opt}}^{\bar{K}}=-200 \mathrm{MeV}$ we have the problem that the optical potential of the kaon turns out to be attractive while experiment tells us that it is repulsive. Also the low density theorem is not fulfilled in our model.

Hence, we discuss in the following the KN-scattering lengths, try to fix our parameters to the KN-scattering lengths and perform a G-parity transformation afterwards to get the $\bar{K} \mathrm{~N}$-case. The isospin averaged scattering length in the tree approximation reads

$$
\bar{a}_{0}=\frac{1}{4} a_{0}^{I=0}+\frac{3}{4} a_{0}^{I=1}=\frac{m_{K}}{4 \pi\left(1+m_{K} / m_{N}\right)}\left(\frac{g_{\sigma K} g_{\sigma N}}{m_{\sigma}^{2}}-2 \frac{g_{\omega K} g_{\omega N}}{m_{\omega}^{2}}\right)=-0.255 \mathrm{fm}
$$

where the experimental value has been taken from [32]. It can be used to fix $g_{\sigma K}$ for known $g_{\omega K}$. Nevertheless, the presented Lagrangian is not able to get the correct scattering length for $\mathrm{KN}$-scattering in the two isospin channels. A way out is, in addition to the $\rho$-meson, to introduce an additional coupling of the kaon to the scalar isovector meson $a_{0}$ which we will denote in the following as $\delta$. The $\mathrm{KN}$-scattering lengths for a given Isospin $I$ on the tree level are then given by

$$
\begin{aligned}
& a_{0}^{I=1}=\frac{m_{K}}{4 \pi\left(1+m_{K} / m_{N}\right)}\left(\frac{g_{\sigma K} g_{\sigma N}}{m_{\sigma}^{2}}+\frac{g_{\delta K} g_{\delta N}}{m_{\delta}^{2}}-2 \frac{g_{\omega K} g_{\omega N}}{m_{\omega}^{2}}-2 \frac{g_{\rho K} g_{\rho N}}{m_{\rho}^{2}}\right) \\
& a_{0}^{I=0}=\frac{m_{K}}{4 \pi\left(1+m_{K} / m_{N}\right)}\left(\frac{g_{\sigma K} g_{\sigma N}}{m_{\sigma}^{2}}-3 \frac{g_{\delta K} g_{\delta N}}{m_{\delta}^{2}}-2 \frac{g_{\omega K} g_{\omega N}}{m_{\omega}^{2}}+6 \frac{g_{\rho K} g_{\rho N}}{m_{\rho}^{2}}\right) .
\end{aligned}
$$

The importance of the $\delta$-meson exchange contribution can be seen if one first sets it to zero and take $\mathrm{SU}(6)$-symmetry for the vector coupling constants. Then the $a_{0}^{I=0}$ scattering length depends only on the term coming from the $\sigma$-meson exchange as $g_{\omega K} g_{\omega N}=3 g_{\rho K} g_{\rho N}$. This gives a large positive scattering length whereas experiment tells us that $a_{0}^{I=0}=-0.09 \mathrm{fm}$ is slightly negative [32]. Taking $g_{\sigma N}=10, g_{\omega N}=13$ as standard values for the RMF model (see table 1) one gets $a_{0}^{I=0} \approx 0.4 \mathrm{fm}$ without the $\delta$-meson contribution. Including the $\delta$-meson term and using $g_{\delta N}=5.95$ from the Bonn model [20] one can fit both scatterings lengths nicely for 


$$
g_{\sigma K} \approx 1.9-2.3, \quad g_{\delta K} \approx 5.6-6.4
$$

for the various parameter sets used. A complete list of the coupling constants of the kaon can be found in table 2. Note that the values are surprisingly close to the one expected from the simple quark-model $\left(g_{\sigma K}=g_{\sigma N} / 3 \approx 3.3, g_{\delta K}=g_{\delta N}=5.95\right)$. The effects of the $\delta$-meson on the properties of neutron star matter were discussed in the previous section and seemed to be quite insigificant. Hence, despite of the rather large coupling constant to the $\delta$-meson its influence on the kaon effective energy in neutron star matter will be rather small compared with the other isoscalar fields.

In addition, the importance of off-shell corrections has been pointed out in detail in [33]. The scalar term has to change sign when going from on-shell $\omega_{K}=m_{K}$ to the off-shell point $\omega_{K}=0$. In the one-boson exchange picture this can be incorporated by adding the following terms

$$
\mathcal{L}_{K}^{\prime \prime}=D_{\mu}^{*} \bar{K} D^{\mu} K\left(\frac{f_{\sigma K} \sigma}{m_{K}}+\frac{f_{\sigma^{*} K} \sigma^{*}}{m_{K}}\right)+D_{\mu}^{*} \bar{K} \tau D^{\mu} K \frac{f_{\delta K} \vec{\delta}}{m_{K}} .
$$

The on-shell constraints eqs. (32) and (33) are still fulfilled if one replaces $g_{\sigma K}$ by $g_{\sigma K}-f_{\sigma K}$ and $g_{\delta K}$ by $g_{\delta K}-f_{\delta K}$, respectively, for the values given in (34). The off-shell behaviour of the scalar contribution is now proportional to

$$
\propto\left(1-\frac{f_{\sigma K}}{g_{\sigma K}} \frac{\omega_{K}^{2}}{m_{K}^{2}}\right) \frac{g_{\sigma K} g_{\sigma N}}{m_{\sigma}^{2}} m_{K}
$$

and changes sign for $\omega=m_{K} / \sqrt{2}$, as it should be [33], if one takes $f_{\sigma K}=2 g_{\sigma K}$. Hence, the decrease of the effective mass of the kaon in the nuclear medium is reduced by these new terms

$$
m_{K}^{* 2}=\frac{m_{K}^{2}+g_{\sigma K} \sigma m_{K}}{1+f_{\sigma K} \sigma / m_{K}}
$$

and makes kaon condensation even less favourable at higher densities (note that both the $\sigma$-field and the coupling constants are negative here).

We have found that the fit based on the adjustment to the KN-scattering lengths leads to an optical potential around $U_{\mathrm{opt}}^{\bar{K}}=-(130 \div 150) \mathrm{MeV}$ at normal nuclear density for the parameter sets used. This is between the two families of solutions found in [27]. 
The general expression for the effective energy of the charged kaon and the antikaon in neutron star matter is given by

$$
\omega_{K^{+}, K^{-}}=m_{K} \sqrt{\frac{m_{K}+g_{\sigma K} \sigma+g_{\sigma^{*} K} \sigma^{*} \pm g_{\delta K} \delta}{m_{K}+2\left(g_{\sigma K} \sigma+g_{\sigma^{*} K} \sigma^{*} \pm g_{\delta K} \delta\right)}} \pm\left(g_{\omega K} V_{0}+g_{\phi K} \phi_{0}+g_{\rho K} R_{0,0}\right)
$$

and shown in fig. 8 for the parameter sets TM1 and TM2 and model 2 including the $\delta$ meson. The two cases (with and without the off-shell terms) are plotted. The energy of the kaon is first increasing due to the low density theorem. The energy of the antikaon is decreasing steadily. After hyperons are present the situation changes dramatically. The potential coming from the $\phi$-field cancels the contribution coming from the $\omega$-meson. Hence, at a certain density the energies of the kaons and antikaons are equal to the effective mass of the kaon, i.e. the curves for kaons and antikaons are crossing at sufficent high densities and the energy of the kaon gets even lower than that of the antikaon! The difference between the two parameter sets considered is not as important as the inclusion of the off-shell terms. These terms effectively shifts the effective mass of the kaon to higher values. Therefore the in-medium energy of both kaons and anitkaons is shifted up to at most $100 \mathrm{MeV}$. The minimum energy of the antikaon reads about $270 \mathrm{MeV}$ for the on-shell and $350 \mathrm{MeV}$ for the off-shell case. As the chemical potential never reaches values above $160 \mathrm{MeV}$ here (or $200 \mathrm{MeV}$ without the $\delta$-meson) antikaon condensation does never occur. We have checked the possibility of antikaon condensation for all parameter sets for model 1 and model 2 and found that at least $100 \mathrm{MeV}$ are missing for the onset of condensation. The most uncertain

term for the effective energy of the antikaon is the one coming from the $\sigma^{*}$-exchange. If one arbitrarily doubles the coupling constant this would lead at maximum to an additional attraction of about $70-80 \mathrm{MeV}$ and still no antikaon condensation is possible within our approach.

\section{CONCLUSION AND OUTLOOK}

We have studied the equation of state of neutron star matter within the relativistic mean field model including hyperons. We have used a representative set of parameters fitted to 
the properties of finite nuclei and included the rather strong hyperon-hyperon interaction induced by additional (hidden) strange meson fields. The coupling constants of the hyperons have been fixed by SU(6)-symmetry relations and hypernuclear properties. Standard parametrizations with different forms of the scalar selfinteraction yield negative values for the nucleon effective mass solely due to the abundant presence of hyperons at high densities. The vector selfinteraction terms and the additional meson exchanges together eliminate this behaviour. According to our calculations, the baryon effective masses become small in neutron star matter at very high densities $\left(\rho>6 \rho_{0}\right)$ and the abundances of the different species are determined by their spin-degeneracy factors (i.e. isospin-saturated matter). In all cases the hyperons get into the game at quite low densities, around $2-3 \rho_{0}$, and the composition is quite similar for all parameter sets studied up to $4 \rho_{0}$. The effects coming from the inclusion of a scalar isovector meson $a_{0}(980)$ have been discussed. The equation of state does not change considerably but all the baryons get now different effective masses. The protons and electrons are getting a little bit suppressed at lower densities and the elctrochemical potential decreases while the $\Sigma^{-}$'s occur at slightly lower densitites compared to the model without the $\delta$-meson contribution.

The possibility of antikaon condensation has been also studied in the framework of the relativistic mean-field model. Fixing the vector coupling constants by SU(3)-constraints, the in-medium energy of the antikaon was calculated for different choices of the optical potential at normal nuclear density. The existence of hyperons, especially the repulsive contribution from $\phi$-exchange and the decrease of the electrochemical potential, makes a antikaon condensed phase less favourable even at very high densities. We get different answers for different parameter sets whether or not antikaons are present in the dense interior of neutron stars. Nevertheless, we see no onset of antikaon condensation below $\rho<6 \rho_{0}$ for all cases studied. Note that the maximum density of neutron star will set another limit for the kaon condensed phase which has not been studied here.

In a second approach we fix the coupling constants to the available KN-scattering data and take care of the off-shell behaviour of the kaon energy. Hence, this approach fulfills the 
low-density theorem for kaons. The effective energy of the kaons and antikaons are dictated by the behaviour of the effective mass of the kaon as the contributions from the vector fields cancel each other. For very high densities $\left(\rho>7 \rho_{0}\right)$ it is possible that the effective energy of the kaon is even lower than that of the antikaon as the contribution form the (hidden) strange meson field exceds the one coming from the $\omega$-meson field. The effective energy of the antikaon turns upward again at this density. Hence, antikaon condensation can never occur in any of our parametrizations as the electrochemical potential is always lower than the minimum energy of the antikaon.

The extrapolation to hyperon-rich matter is associated with large uncertainties due to the unknown hyperon-kaon interaction (e.g. $\bar{K} \Xi$-interaction). The effective energy of the kaon is very much influenced by large cancellation effects coming from the vector fields. A slight increase of the coupling constant might therefore change the antikaon energy by 100 $\mathrm{MeV}$ which are at least missing for the onset of kaon condensation. Hence, there might be still some niches for antikaon condensation. Nevertheless, the overall trend is quite clear: the presence of hyperons makes the onset of antikaon condensation quite unlikely to happen.

The vector selfinteraction terms as well as the hyperon-hyperon interactions might influence also the maximum mass, the rotational frequency and cooling properties of neutron stars. These questions will be studied in a forthcoming work.

\section{ACKNOWLEDGEMENTS}

We would like to thank J. Bondorf, N.K. Glendenning, V. Thorsson, F. Weber, W. Weise and Th. Waas for useful discussions and remarks. This work is supported in part by the International Science Foundation (Soros) grant N8Z000 and EU-INTAS grant 943405. J. Schaffner is supported by the EU-program 'Human Capital and Mobility' (contract ERBCHBGCT930407). The authors also thank the Niels Bohr Institute for kind hospitality and financial support. 


\section{REFERENCES}

[1] N.K. Glendenning: Phys. Lett. 114B, 392 (1982); Astrophys. J. 293, 470 (1985); Z. Phys. A326, 57 (1987);

F. Weber and N.K. Glendenning: Proc. of the Int. Summer School on Nuclear Astrophysics, Tianjin, P.R. China, World Scientific, 1991, p. 64-183

[2] P.-G. Reinhard: Z. Phys. A329, 257 (1988)

[3] A.R. Bodmer: Nucl. Phys. A526, 703 (1991)

[4] Y. Sugahara and H. Toki: Nucl. Phys. A579, 557 (1994)

[5] S. Gmuca: J. Phys. G17, 1115 (1991); Z. Phys. A342, 387 (1991); Nucl. Phys. A547, $447(1992)$

[6] J. Schaffner, C.B. Dover, A. Gal, C. Greiner, H. Stöcker: Phys. Rev. Lett. 71, 1328 $(1993)$

[7] J. Schaffner, C.B. Dover, A. Gal, D.J. Millener, C. Greiner, H. Stöcker: Ann. of Phys. (N.Y.) 235, 35 (1994)

[8] D.B. Kaplan and A.E. Nelson: Phys. Lett. B175, 57 (1986)

A.E. Nelson and D.B. Kaplan: Phys. Lett. B192, 193 (1987)

[9] G.E. Brown, K. Kubodera, M. Rho, V. Thorsson: Phys. Lett. B291, 355 (1992)

V. Thorsson, M. Prakash, J.M. Lattimer: Nucl. Phys. A572, 693 (1994)

[10] G.E. Brown, C.-H. Lee, M. Rho, V. Thorsson: Nucl. Phys. A567, 937 (1994)

[11] T. Muto: Prog. Theor. Phys. 89, 415 (1993)

[12] P.J. Ellis, R. Knorren, M. Prakash: Phys. Lett. B349, 11 (1995)

[13] J. Boguta and A.R. Bodmer: Nucl. Phys. A292, 413 (1977)

J. Boguta and H. Stöcker: Phys. Lett. B120, 289 (1983) 
[14] M. Rufa, P.-G. Reinhard, J. Maruhn, W. Greiner, M.R. Strayer: Phys. Rev. C38, 390 (1988)

[15] M.M. Sharma, M.A. Nagarajan, P. Ring: Phys. Lett. B312, 377 (1993)

[16] R. Brockmann, W. Weise: Phys. Lett. 69B, 167 (1977)

J. Boguta, S. Bohrmann: Phys. Lett. 102B, 93 (1981)

M. Rufa, H. Stöcker, P.-G. Reinhard, J. Maruhn, W. Greiner: J. Phys. G13, L143 (1987)

J. Mareš, J. Žofka: Z. Phys. A333, 209 (1989)

M. Rufa, J. Schaffner, J. Maruhn, H. Stöcker, W. Greiner, P.-G. Reinhard: Phys. Rev. C42, 2469 (1990)

[17] N.K. Glendenning and S.A. Moszkowski: Phys. Rev. Lett. 67, 2414 (1991)

[18] J. Schaffner, C. Greiner, H. Stöcker: Phys. Rev. C46, 322 (1992)

[19] J.M. Lattimer, C.J. Pethick, M. Prakash, P. Haensel: Phys. Rev. Lett. 66, 2701 (1991)

[20] R. Machleidt, K. Holinde, C. Elster: Phys. Rep. 149, 1 (1987)

[21] R. Büttgen, K. Holinde, A. Müller-Groeling, J. Speth, P. Wyborny: Nucl. Phys. A506, $586(1990)$

A. Müller-Groeling, K. Holinde, J. Speth: Nucl. Phys. A513, 557 (1990)

[22] M. Lutz, A. Steiner, W. Weise: Phys. Lett. B278, 29 (1992); Nucl. Phys. A547, 755 (1994)

[23] C.-H. Lee, H. Jung, D.-P. Min, M. Rho: Phys. Lett. B326, 14 (1994) and references therein

[24] J. Schaffner, A. Gal, I.N. Mishustin, H. Stöcker, W. Greiner: Phys. Lett. B334, 268 (1994)

[25] C.-H. Lee, G.E. Brown, D.-P. Min, M. Rho: Nucl. Phys. A585, 401 (1995) 
[26] T. Maruyama, H. Fujii, T. Muto, T. Tatsumi: Phys. Lett. B337, 19 (1994)

[27] E. Friedman, A. Gal, C.J. Batty: Phys. Lett. B308, 6 (1993); Nucl. Phys. A579, 518 (1994)

[28] P.B. Siegel and W. Weise: Phys. Rev. C38, 2221 (1988)

[29] V. Koch: Phys. Lett. B337, 7 (1994)

[30] N. Kaiser, P.B. Siegel, W. Weise: Technische Universität München preprint TUM/T3995-5, March 1995

[31] T.A. Armstrong et al., WA76 Collaboration: Z. Phys. A51, 351 (1991)

[32] T. Barnes and E.S. Swanson: Phys. Rev. C49, 1166 (1994)

[33] J. Delorme, M. Ericson, T.E.O. Ericson: Phys. Lett. B291, 379 (1992)

H. Yabu, S. Nakamura, K. Kubodera: Phys. Lett. B317, 269 (1993)

H. Yabu, S. Nakamura, F. Myhrer, K. Kubodera: Phys. Lett. B315, 17 (1993) 


\section{TABLES}

TABLE I. The coupling constants of the parameter sets used. The vector coupling constants for the hyperons are be taken from $\mathrm{SU}(6)$-relations. The nucleons do not couple to the $\sigma^{*}$ - and the $\phi$-meson $\left(g_{\sigma^{*} N}=g_{\phi N}=0\right)$. The $\Lambda$ does not couple to the isovector fields $\left(g_{\rho \Lambda}=0\right)$. The coupling constants for the $\Sigma$ 's are the same as for the $\Lambda$ except for the isovector coupling constant which is $g_{\rho \Sigma}=2 g_{\rho N}$. The parameters for the scalar and vector selfinteraction terms are not given, they can be found in the corresponding references.

\begin{tabular}{|c|c|c|c|c|c|c|}
\hline Set & nl-z & nl-sh & pl-z & pl-40 & tm1 & $\operatorname{tm} 2$ \\
\hline Ref. & 14 & 15 & [2] & 2 & 幽 & 4 \\
\hline$g_{\sigma N}$ & 10.0553 & 10.4440 & 10.4262 & 10.0514 & 10.0289 & 11.4694 \\
\hline$g_{\omega N}$ & 12.9086 & 12.9450 & 13.3415 & 12.8861 & 12.6139 & 14.6377 \\
\hline$g_{\rho N}$ & 4.8494 & 4.3830 & 4.5592 & 4.8101 & 4.6322 & 4.6783 \\
\hline$g_{\sigma \Lambda}$ & 6.23 & 6.47 & 6.41 & 6.20 & 6.21 & 7.15 \\
\hline$g_{\omega \Lambda}$ & 8.61 & 8.63 & 8.89 & 8.59 & 8.41 & 9.76 \\
\hline$g_{\sigma^{*} \Lambda}$ & 6.77 & 6.85 & 6.93 & 6.78 & 6.67 & 7.65 \\
\hline$g_{\phi \Lambda}$ & -6.09 & -6.10 & -6.29 & -6.07 & -5.95 & -6.90 \\
\hline$g_{\sigma \Xi}$ & 3.45 & 3.59 & 3.52 & 3.43 & 3.49 & 3.94 \\
\hline$g_{\omega \Xi}$ & 4.30 & 4.31 & 4.45 & 4.30 & 4.20 & 4.88 \\
\hline$g_{\rho \Xi}$ & 4.85 & 4.38 & 4.56 & 4.81 & 4.63 & 4.68 \\
\hline$g_{\sigma^{*} \Xi}$ & 12.59 & 12.66 & 12.95 & 12.57 & 12.35 & 14.18 \\
\hline$g_{\phi \Xi}$ & -12.17 & -12.20 & -12.58 & -12.15 & -11.89 & -13.80 \\
\hline
\end{tabular}


TABLE II. The coupling constants for the kaons. The coupling constants to the $\sigma$ - and $\delta$-meson are fixed by the s-wave KN-scattering lenghts. The vector coupling constants are chosen from $\mathrm{SU}(3)$-relations. The coupling constant to the $\sigma^{*}$-meson is taken from $f_{0}$-decay [31].

\begin{tabular}{|c|c|c|c|c|c|c|}
\hline Set & nl-z & nl-sh & pl-z & pl-40 & tm1 & $\operatorname{tm} 2$ \\
\hline Ref. & 14 & 15 & 2] & 2 & (4) & (4) \\
\hline$g_{\sigma K}$ & 1.85 & 2.05 & 2.20 & 2.27 & 1.93 & 2.27 \\
\hline$g_{\omega K}$ & 3.02 & 3.02 & 3.02 & 3.02 & 3.02 & 3.02 \\
\hline$g_{\rho K}$ & 3.02 & 3.02 & 3.02 & 3.02 & 3.02 & 3.02 \\
\hline$g_{\sigma^{*} K}$ & 2.65 & 2.65 & 2.65 & 2.65 & 2.65 & 2.65 \\
\hline$g_{\phi K}$ & 4.27 & 4.27 & 4.27 & 4.27 & 4.27 & 4.27 \\
\hline$g_{\delta K}$ & 6.37 & 5.59 & 5.89 & 6.31 & 5.87 & 5.94 \\
\hline
\end{tabular}




\section{FIGURES}

FIG. 1. The composition of neutron star matter with hyperons in model 2 using parameter set PL-Z. The jump in the curves is due to the negative effective mass of the nucleon.

FIG. 2. The composition of neutron star matter with hyperons in model 2 using parameter set TM1 with vector selfinteraction terms. The matter approaches isospin-saturation at high densities.

FIG. 3. The effective masses of the baryons versus the density for model 2 using set TM1. All effective masses remain positive for the density range considered.

FIG. 4. The field potentials and the electrochemical potential versus the density for model 2 using set TM1. Quite high values are reached indicating large cancelation effects at high densities.

FIG. 5. The equation of state in model 2 for various parameter sets. The causal limit $p=\epsilon$ is also shown.

FIG. 6. The effective masses of the baryons versus the density for model 2 using set TM1 with $\delta$-mesons. All baryons get a different effective mass due to the additional meson.

FIG. 7. The effective energy minus the chemical potential of the $K^{-}$over the density for model 2 using various parameter sets. The upper curves are calculated for an optical potential of $U_{\mathrm{opt}}^{\bar{K}}=-120 \mathrm{MeV}$, the lower ones for $U_{\mathrm{opt}}^{\bar{K}}=-200 \mathrm{MeV}$.

FIG. 8. The effective energy of the kaon and the antikaon for model 2 with $\delta$-mesons using set TM1. The electrochemical potential is also plotted. Antikaon condensation does not occur over the whole density region considered. 


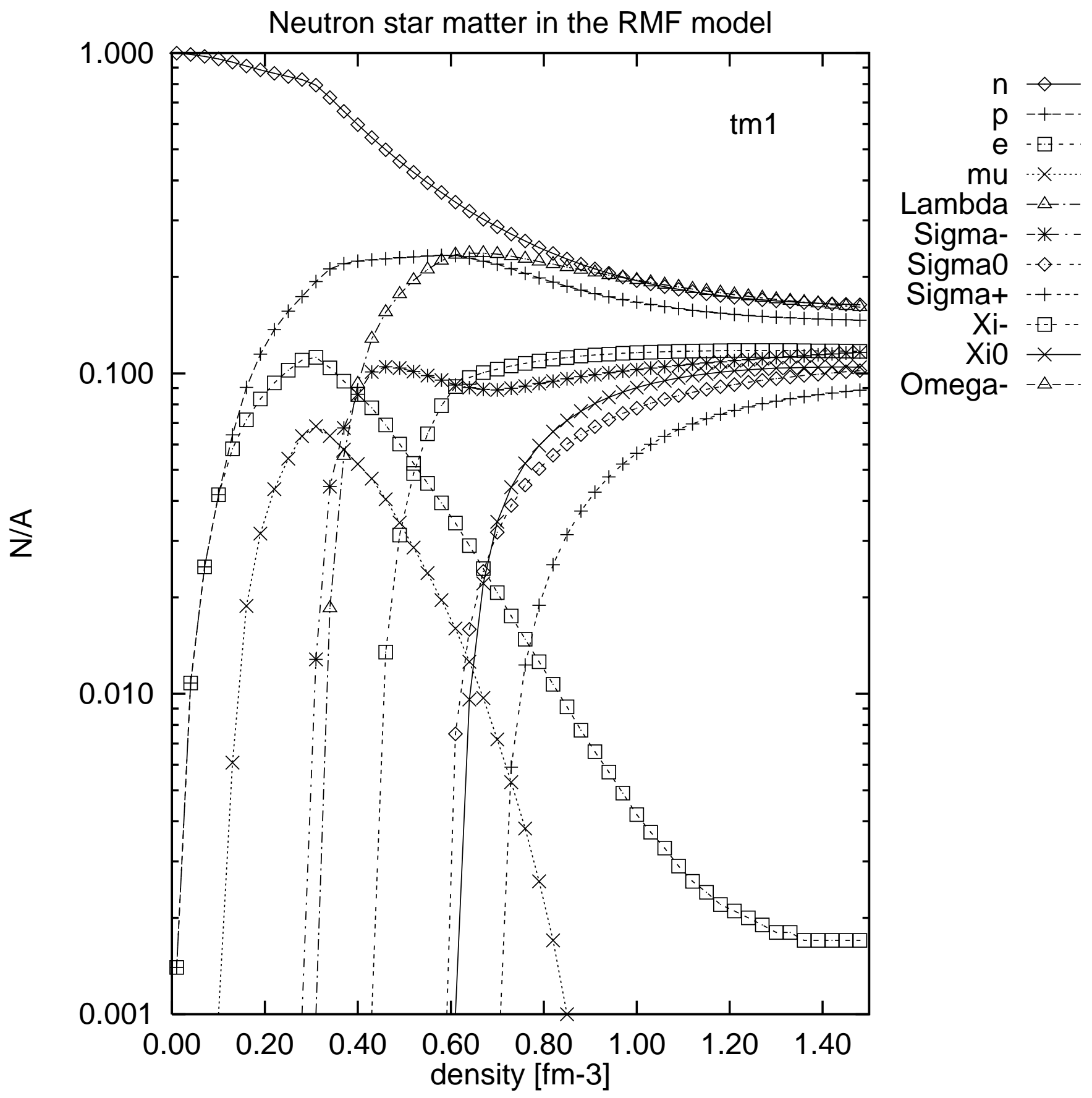




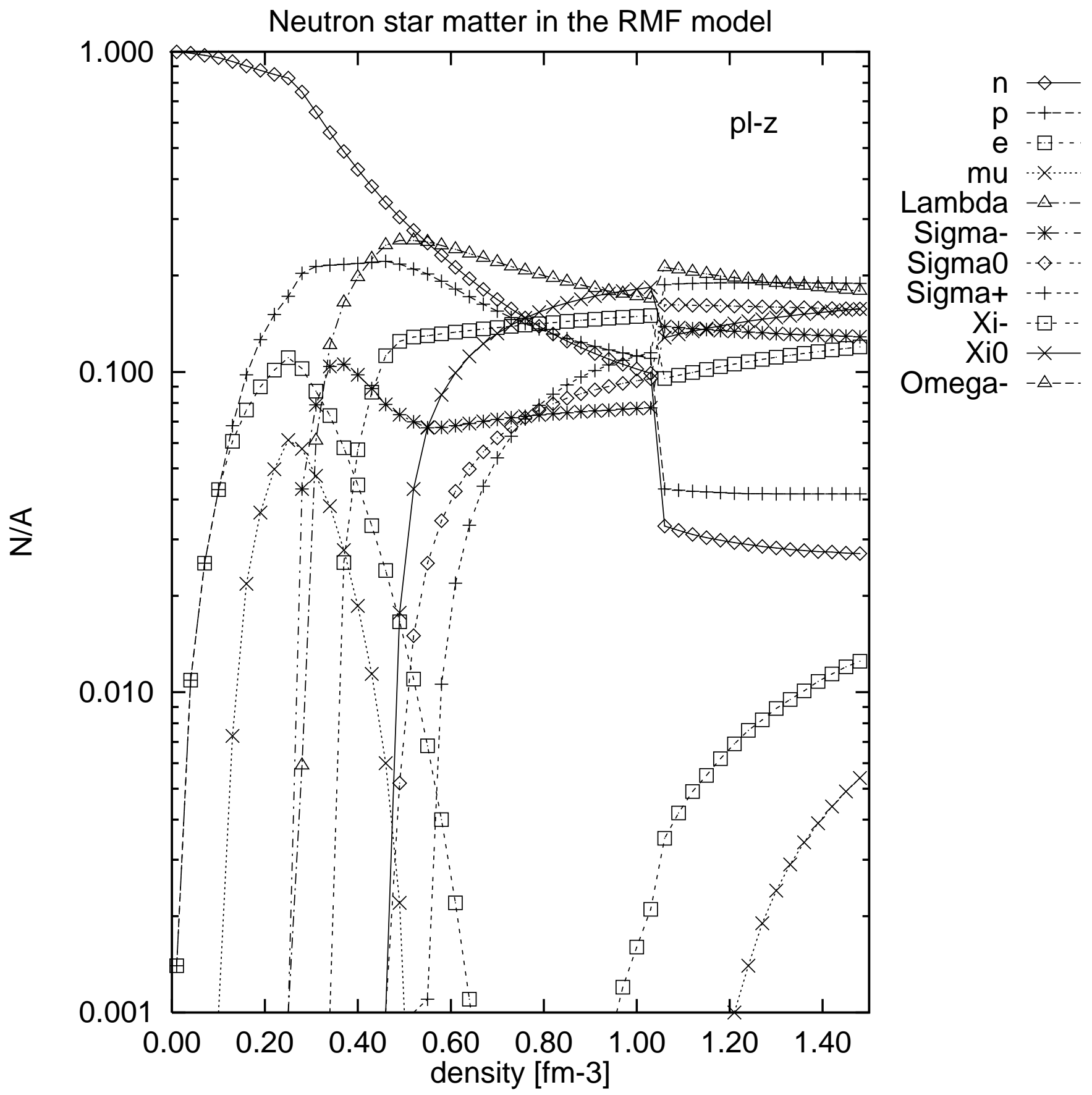




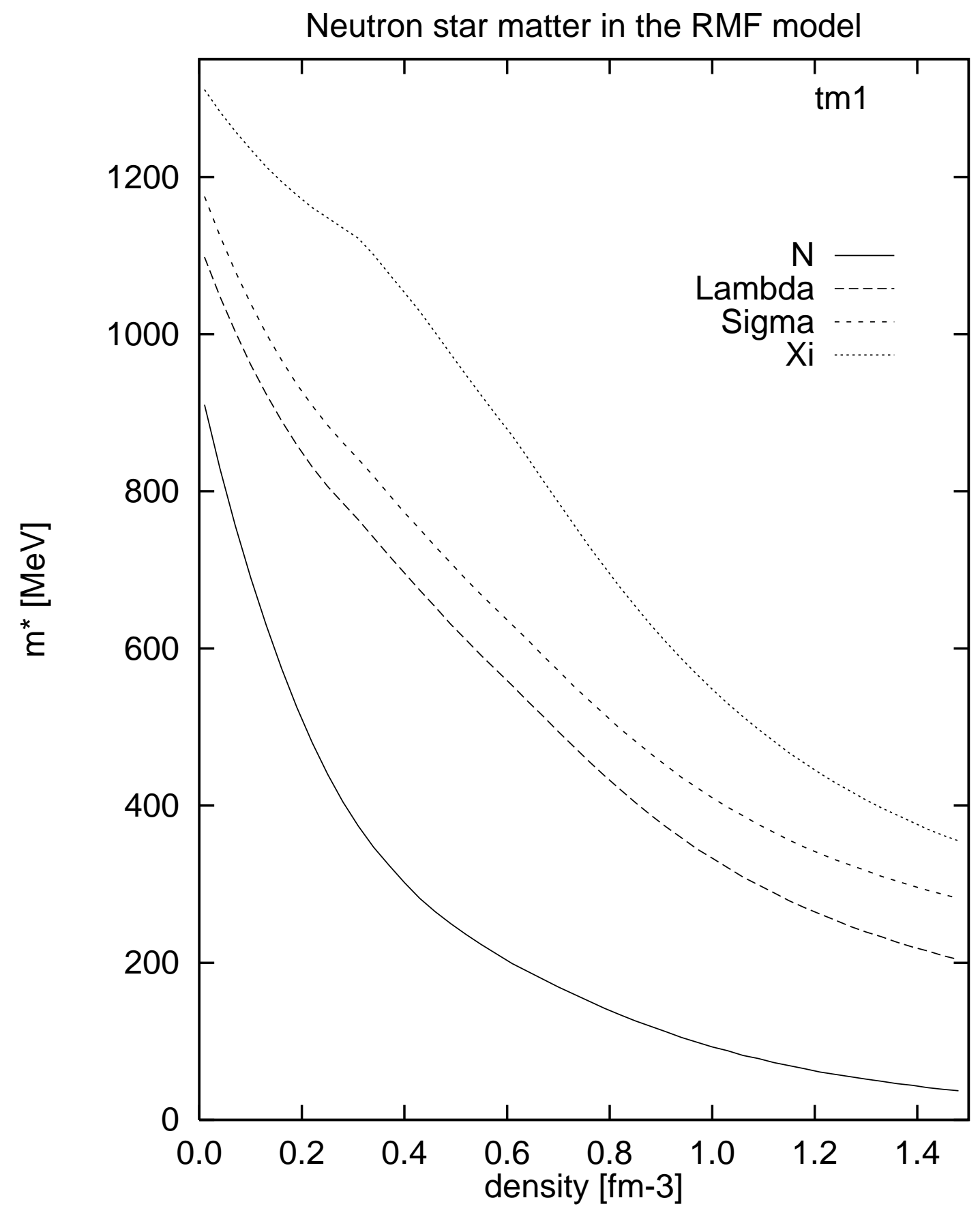




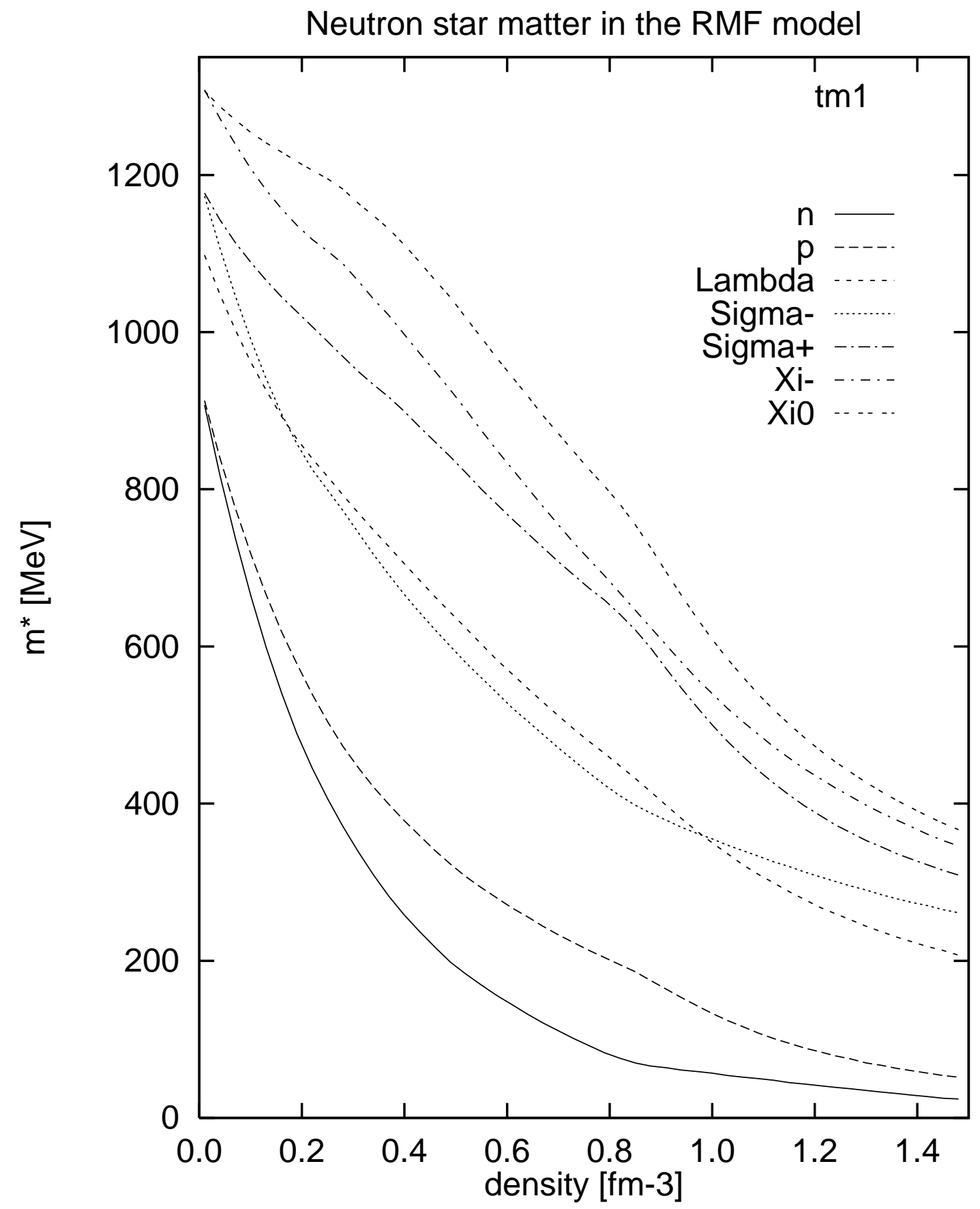




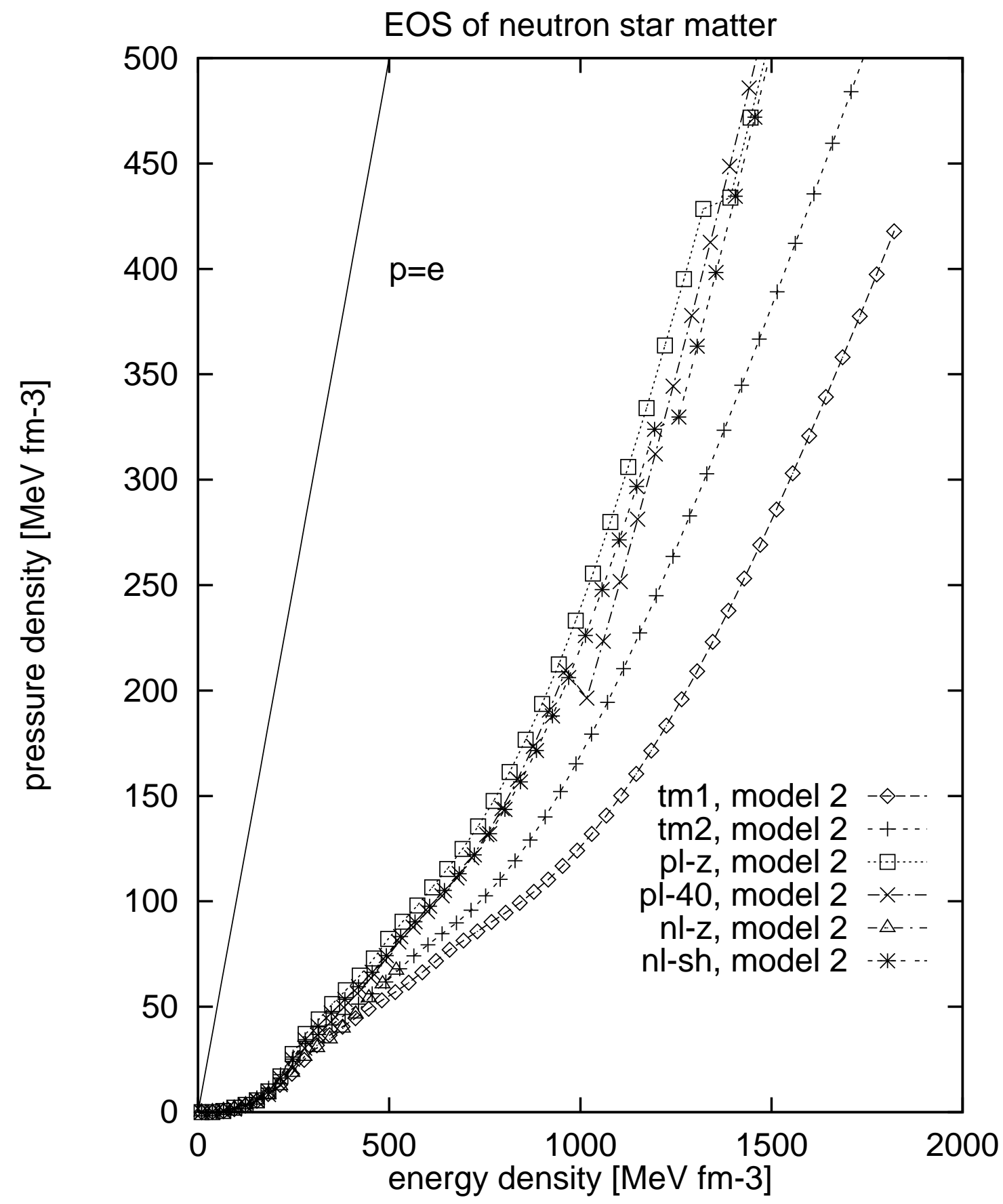




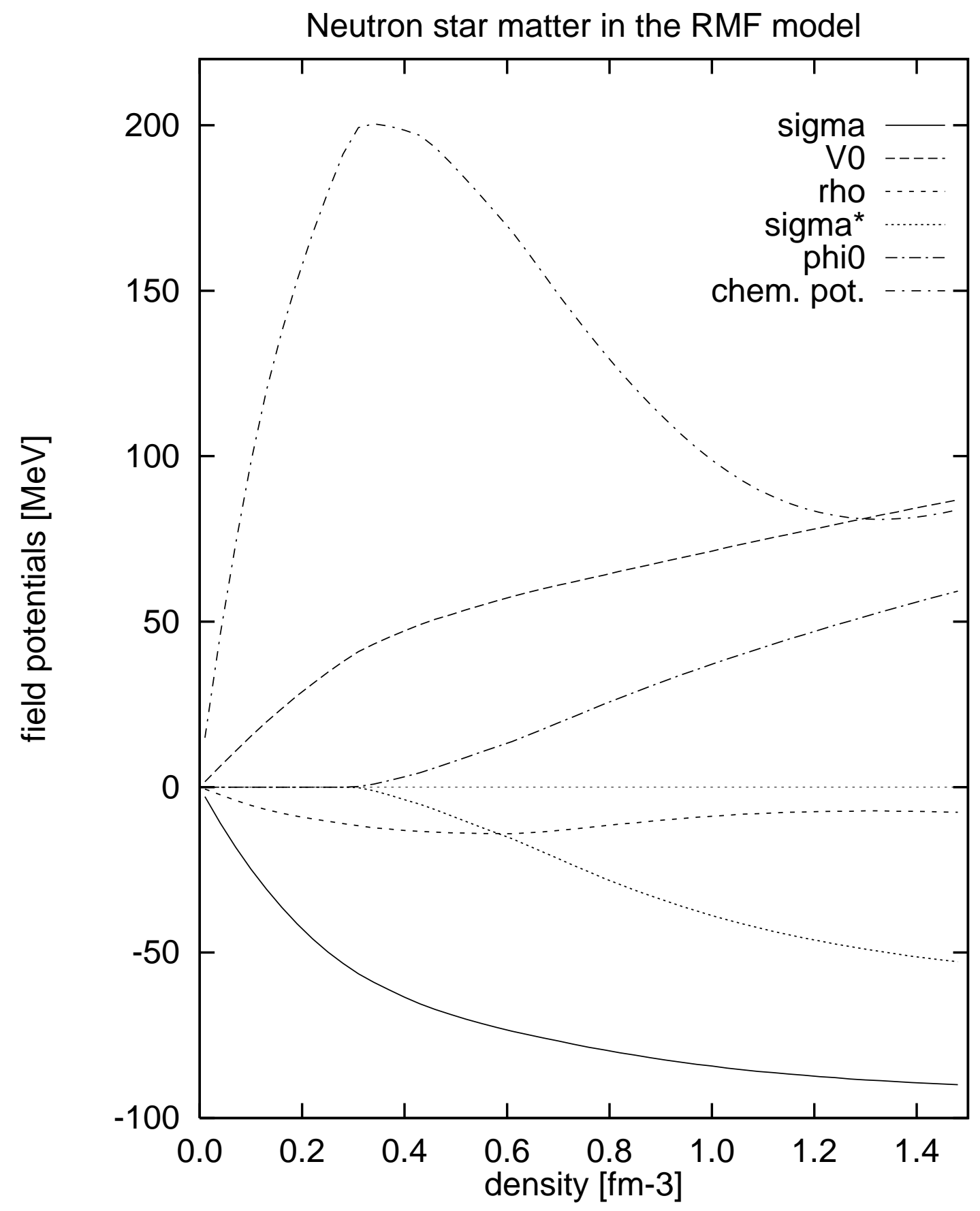




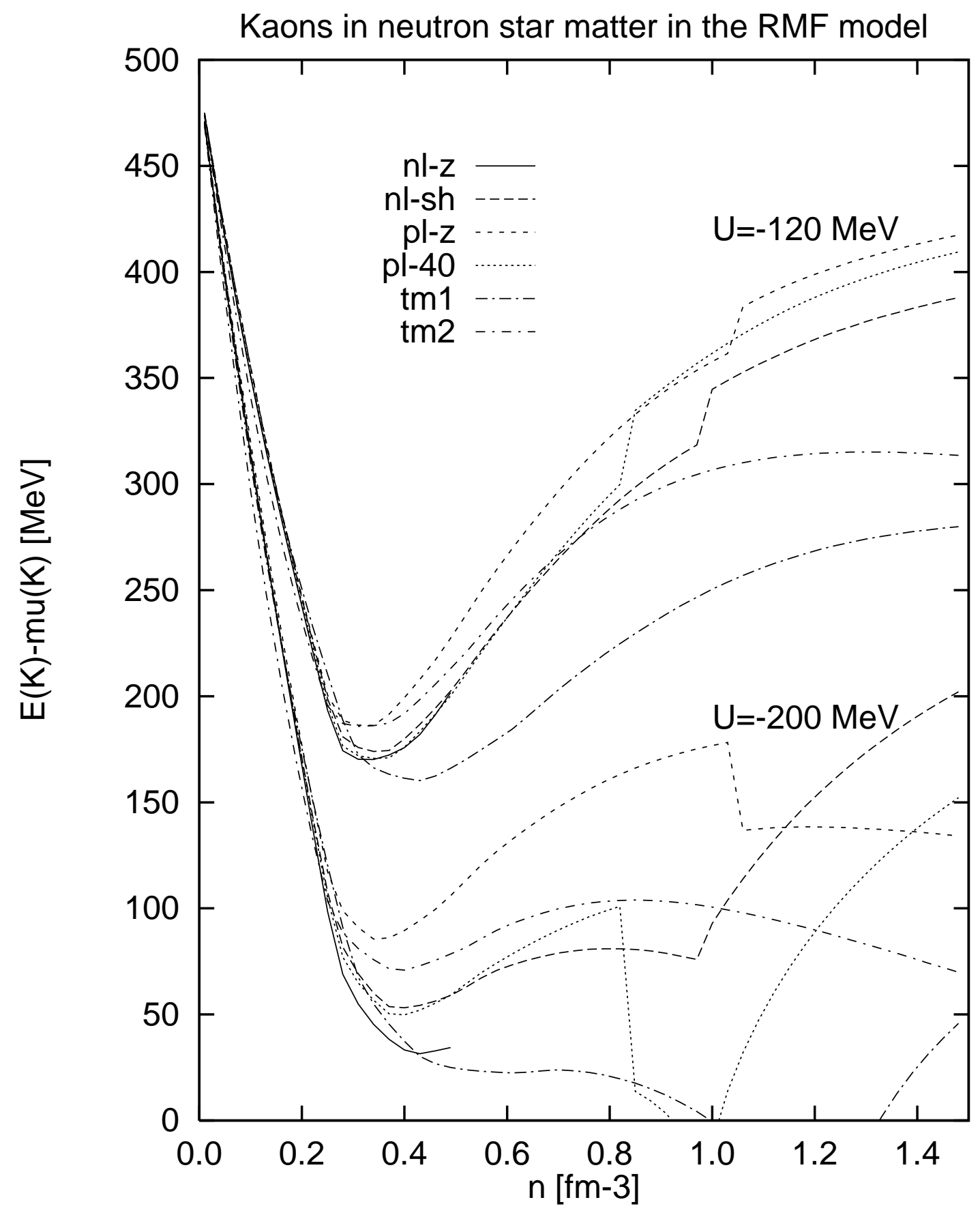




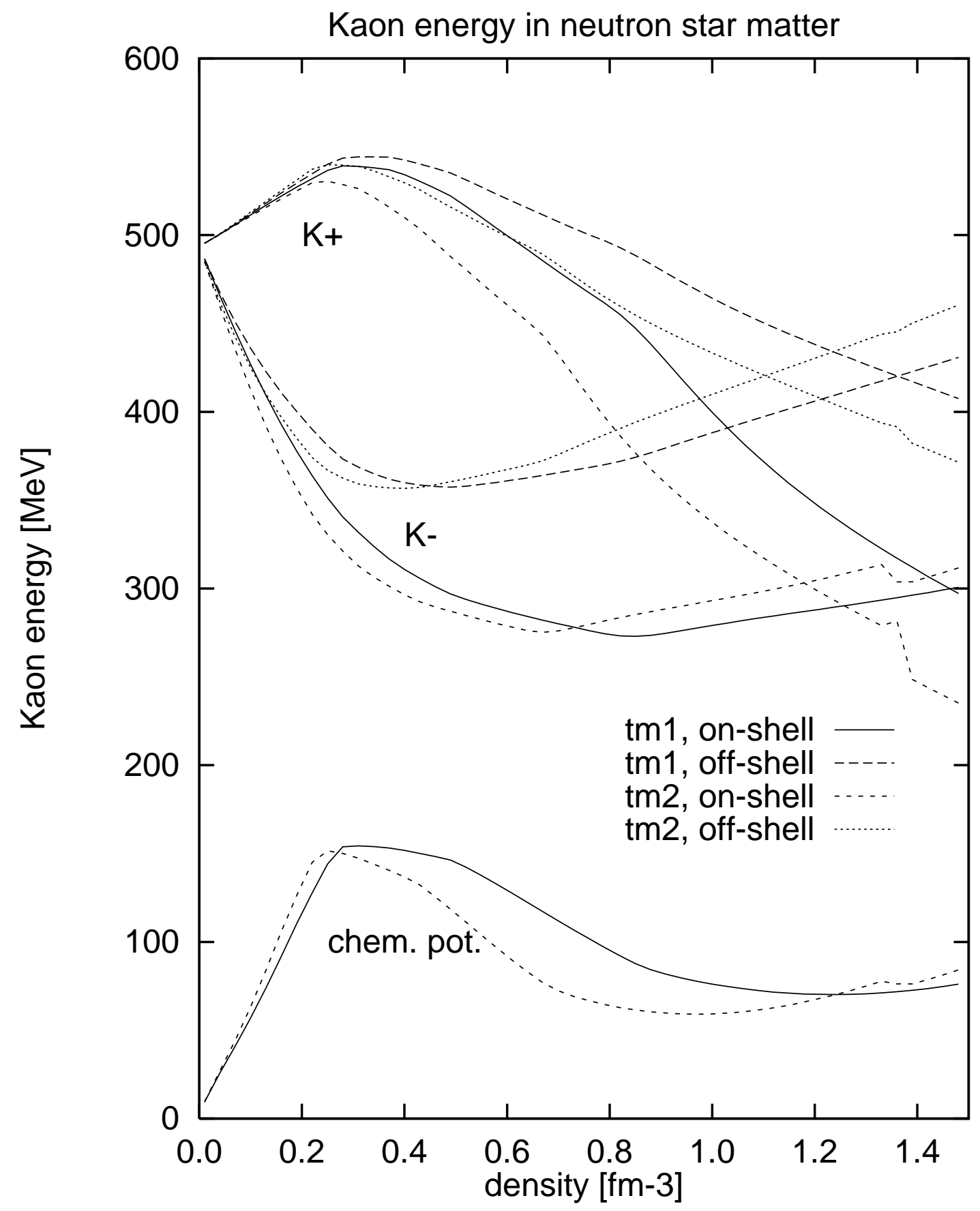

